\title{
APPROXIMATION BY RATIONAL FUNCTIONS ON RIEMANN SURFACES
}

\author{
M. GOLDSTEIN ${ }^{1}$ AND J. L. WALSH ${ }^{2}$
}

\begin{abstract}
In this paper, we show that if $F \in L^{p}(k, \alpha)$ on $\Gamma$ where $\Gamma$ denotes the border of a compact bordered Riemann surface $\bar{R}$, then $F$ can be uniquely written as the sum of a function in $H^{p}(k, \alpha)$ and a function in $G^{p}(k, \alpha)$ and moreover that $F$ can be approximated on $\Gamma$ in $L^{p}$ norm to within $A / n^{k+\alpha}$ by a sequence of rational functions on the union of $\bar{R}$ with its double.
\end{abstract}

The purpose of this paper is to generalize some results of the second named author [4] concerning the Hardy classes $H^{p}$ and their particularizations to the classes $H^{p}(k, \alpha)$ of functions satisfying (together with certain derivatives) a Lipschitz condition to Riemann surfaces.

Let $\gamma$ denote the unit circle. We define the class $L^{p}(k, \alpha)(0<\alpha<1$, $1<p<\infty)$ to be the set of functions possessing derivatives up to order $k$ on $\gamma$ whose $k$ th derivatives satisfy a $p$ th mean integrated Lipschitz condition of order $\alpha . H^{p}(k, \alpha)$ will denote the subclass of functions belonging to $L^{p}(k, \alpha)$ which belong to the Hardy class $H^{p}$ of the interior of $\gamma$ while $G^{p}(k, \alpha)$ will denote those functions belonging to $L^{p}(k, \alpha)$ which are boundary functions of functions belonging to $H^{p}$ of the exterior of $\gamma$ and vanishing at $\infty$. In [4], it is shown that every function of the class $L^{p}(k, \alpha)$ can be written uniquely as the sum of a function in $H^{p}(k, \alpha)$ and a function in $G^{p}(k, \alpha)$. It is this result that we shall generalize to the setting of Riemann surfaces and in addition obtain some results on approximation by rational functions.

Let $R$ denote the interior of a compact bordered Riemann surface $\bar{R}$ with boundary $\Gamma$. Suppose $f$ possesses derivatives up to order $k$ on $\Gamma$. Let $\Gamma_{1}, \cdots, \Gamma_{n}$ denote the connected components of $\Gamma$ and let $\chi_{i}$, $i=1, \cdots, n$, denote the uniformizers which map $\Gamma_{i}$ onto $\gamma$. We shall say that $f$ is of class $L^{p}(k, \alpha)$ on $\Gamma$ if $f \circ \chi_{i}^{-1}$ is of class $L^{p}(k, \alpha)$ on $\gamma$ for all $i$. This definition is independent of the choice of uniformizing variables as a consequence of a theorem of Hardy and Littlewood [3].

Received by the editors February 4, 1972.

AMS 1969 subject classifications. Primary 3070; Secondary 3067.

Key words and phrases. Lipschitz condition, Hardy classes, rational functions, meromorphic differential.

1 The author is partially supported by an NSF research associateship.

2 The author is partially supported by USAF grant AF-69-1690.

(c) American Mathematical Society 1973 
Let $\zeta \in R$ and denote by $g_{\zeta}(z)$ the Green's function of $R$ with pole at $\zeta$. Let $\hat{R}$ denote the double of $R$. By the symmetry principle, $g_{\zeta}(z)$ has a meromorphic extension to $\bar{R} \cup \hat{R}$ which we shall continue to denote by $g_{\zeta}(z)$. Let $\delta g_{\zeta}(z)=2\left(\partial g_{\zeta}(z) / \partial z\right) d z$ and let $\partial_{\delta g_{\zeta}(z)}$ denote the divisor of $\delta g_{\zeta}(z)$. Define $H^{p}\left(\hat{R}, \partial_{\delta g_{\zeta}(z)} \mid \hat{R}\right)$ to be the class of meromorphic functions $f$ on $\hat{R}$ which outside of a compact subset of $\hat{R}$ belong to the Hardy class $H^{p}$ and have the property that $\partial_{f}+\partial_{\delta g_{a}} \geqq 0$. Let $H^{p}(R)$ denote the Hardy class of analytic functions on $R$ whose moduli raised to the $p$ th power possess a harmonic majorant. Let $H^{p}(k, \alpha)$ denote the class of functions in $H^{p}(R)$ whose boundary values belong to $L^{p}(k, \alpha)$. Let $G^{p}(k, \alpha)$ denote those functions in $H^{p}\left(\hat{R}, \partial_{\delta g_{\zeta}(z)} \mid \hat{R}\right)$ whose boundary functions belong to $L^{p}(k, \alpha)$.

We shall now state and prove our main theorem which is a generalization of Theorem 4 in [4].

THEOREM 1. Let $F(z) \in L^{p}(k, \alpha)$ on $\Gamma$. Then on $\Gamma$ we may write $F(z) \equiv f(z)+g(z)$ where $f(z) \in H^{p}(k, \alpha)$ and $g(z) \in G^{p}(k, \alpha)$.

Proof. The assumption that $F \in L^{p}(k, \alpha)$ on $\Gamma$ implies that $F$ is of class $L^{p}$ on $\Gamma$ and hence by a theorem of Heins [2, Theorem 8, p. 81] we may write $F(z) \equiv f(z)+g(z)$ where $f \in H^{p}(R)$ and $g \in H^{p}\left(\hat{R}, \partial_{\delta g_{\zeta}(z)} \mid \hat{R}\right)$. Furthermore, $\|f\|_{p},\|g\|_{p} \leqq C\|F\|_{p}$, where $C$ is a constant depending only on $p$. By $\|F\|_{p}$, we mean

$$
\left(\frac{1}{2 \pi} \int_{\Gamma}|F|^{p} \frac{\partial g_{\zeta}(z)}{\partial n} d S\right)^{1 / p} .
$$

Thus it follows from these Riesz type inequalities together with Theorem 1 in [4] that $f$ and $g$ are of class $L^{p}(k, \alpha)$ on $\Gamma$. Hence $f$ and $g$ belong respectively to the classes $H^{p}(k, \alpha)$ and $G^{p}(k, \alpha)$.

Insofar as approximation by rational functions goes, we have the following result.

THEOREM 2. Under the conditions of Theorem 1, there exist rational functions $R_{n}(z)$ on $\bar{R} \cup \hat{R}$ satisfying

$$
\left\|F(z)-R_{n}(z)\right\|_{n} \leqq A / n^{k+\alpha},
$$

and conversely if there exist rational functions $R_{n}(z)$ on $\bar{R} \cup \hat{R}$ which are free from poles on $\Gamma$ and which satisfy the above inequality, then $F \in L^{p}(k, \alpha)$ on $\Gamma$.

Proof. Suppose first of all that $F \in L^{p}(k, \alpha)$. Then by Theorem 1, p. 264 in [4], there exist analytic functions $F_{n}(z)$ in a two-sided neighborhood $\bar{D}$ of $\Gamma$ which we can and will take to be a system of ring domains satisfying on $\Gamma$ the inequality

$$
\left\|F(z)-F_{n}(z)\right\|_{p} \leqq A^{\prime} / n^{k+\alpha}
$$


It follows from a construction given by Behnke and Sommer [1, pp. 583-584] that there exists a meromorphic differential $d w(\zeta, z)$ on $\bar{R} \cup \hat{R}$ which for fixed $z$ is a differential in $\zeta$ which on $\bar{R}$ is analytic except for a simple pole at $z$ with residue 1 while for fixed $\zeta, w(\zeta, z)$ is a meromorphic function on $\bar{R} \cup \hat{R}$ which is free from poles on $\Gamma$. By taking $\bar{D}$ so small that for $\zeta \in \bar{D}, \zeta \neq z, d w(\zeta, z)$ for fixed $z$ is an analytic differential in $\bar{D}$, we have by the residue theorem $[1$, p. 545] that, for $z \in \Gamma$,

$$
F_{n}(z)=\frac{1}{2 \pi i} \int_{\partial D} F_{n}(\zeta) d w(\zeta, z)
$$

We now approximate the integral by a sum and thus obtain the existence of rational functions on $\bar{R} \cup \hat{R}$ satisfying the inequality

$$
\left\|F_{n}(z)-R_{n}(z)\right\|_{p} \leqq 1 / n^{k+\alpha} .
$$

Combining this with the first inequality, we have on taking $A=A^{\prime}+1$ the desired conclusion. To obtain the converse, we have merely to apply Theorem 1 in [4].

It is interesting to observe that if we apply Heins' theorem to the rational functions $R_{n}(z)$, we obtain the existence of functions $p_{n}(z) \in H^{p}(R)$ and $q_{n}(z) \in H^{p}\left(\hat{R}, \partial_{\delta g_{\zeta}(z)} \mid \hat{R}\right)$ such that on $\Gamma$,

$$
\left\|f(z)-p_{n}(z)\right\|_{p} \leqq A / n^{k+\alpha}, \quad\left\|g(z)-q_{n}(z)\right\|_{p} \leqq \dot{A} / n^{k+\alpha} .
$$

\section{REFERENCES}

1. H. Behnke and F. Sommer, Theorie der analytischen Funktionen einer komplexen Veränderlichen, Die Grundlehren der math. Wissenschaften, Band 77, Springer-Verlag, Berlin, 1962. MR 26 \#5137.

2. M. Heins, Hardy classes on Riemann surfaces, Lecture Notes in Math., no. 98, Springer-Verlag, Berlin and New York, 1969. MR 40 \#338.

3. J. L. Walsh and H. G. Russell, Integrated continuity conditions and degree of approximation by polynomials or by bounded analytic functions, Trans. Amer. Math. Soc. 92 (1959), 355-370. MR 21 \#7311.

4. J. L. Walsh, Mean approximation by polynomials on a Jordan curve, J. Approximation Theory 4 (1971), 263-268.

Department of Mathematics, University of Maryland, College Park, MARYLAND 20742 